\title{
A synthetic phytosiderophore analog, proline-2'-deoxymugineic acid, is efficiently utilized by dicots
}

\author{
Daisei Ueno (D) Y Yuta Ito $\cdot$ Miho Ohnishi • \\ Chikahiro Miyake $\cdot$ Takayuki Sohtome • \\ Motofumi Suzuki
}

Received: 17 July 2021 / Accepted: 8 September 2021 / Published online: 20 September 2021

(C) The Author(s) 2021

\begin{abstract}
Purpose Phytosiderophores (PS) from grasses solubilize sparingly soluble iron (Fe), and the resultant PS-Fe is an Fe source even for dicots. Recently, the synthetic PS proline-2'-deoxymugineic acid (PDMA) has been developed as a moderately biodegradable $\mathrm{Fe}$ fertilizer for grasses. We aimed to investigate whether PDMA-Fe is also a good Fe source for dicots.

Methods The availability of PDMA-Fe to cucumber was evaluated in a calcareous substrate and hydroponic cultures at $\mathrm{pH} 7.0-9.0$ by determining chlorophyll level, PSII activity, and Fe uptake. EDDHA-Fe,
\end{abstract}

Responsible Editor: Miroslav Nikolic.

Supplementary Information The online version contains supplementary material available at https://doi. org/10.1007/s11104-021-05152-z.

D. Ueno $(\bowtie) \cdot$ Y. Ito

Faculty of Agriculture and Marine Science, Kochi

University, 200 Otsu, Monobe, Nankoku, Kochi 783-8502,

Japan

e-mail: daisei_u@kochi-u.ac.jp

Y. Ito

e-mail: itoyu.golf.1998@gmail.com

M. Ohnishi · C. Miyake

Department of Applied Biological Science, Graduate

School for Agricultural Science, Kobe University, 1-1,

Rokkodai, Nada, Kobe, Hyogo 657-8501, Japan

e-mail: miho.ohnishi02@gmail.com

C. Miyake

e-mail: chikahiro_miyake@outlook.com
EDTA-Fe, and citrate-Fe were used as controls. The reducibility of Fe chelates by roots was measured to determine the mechanism underlying differences in availability. Expressions of $\mathrm{Fe}$ deficiency-inducible genes were analyzed to estimate the $\mathrm{Fe}$ status in plants.

Results The application of PDMA-Fe and EDDHA$\mathrm{Fe}$ to a calcareous substrate reduced Fe-deficient chlorosis to a similar extent; however, the shoot $\mathrm{Fe}$ concentration was higher in the PDMA-Fe treatment. In the hydroponic culture, the availability of PDMA-Fe was higher than that of the other chelates at all $\mathrm{pH}$ levels, and this was confirmed by higher PSII activity and lower expression of Fe deficiencyinducible genes. The reducibility assay revealed that

\author{
M. Ohnishi · C. Miyake \\ Core Research for Environmental Science and Technology \\ (CREST) of Japan Science and Technology Agency, 7, \\ Goban, Chiyoda, Tokyo 102-0076, Japan

\section{T. Sohtome} \\ Research and Development Department, Bunkoukeiki Co., \\ Ltd, 4-8, Takakura, Hachioji, Tokyo 192-0033, Japan \\ e-mail: saotome@bunkoukeiki.co.jp \\ M. Suzuki \\ Aichi Steel Corporation, 1 Wanowari, Arao, \\ Aichi 476-8666 Tokai, Japan \\ e-mail: motofumi-s@he.aichi-steel.co.jp
}


the reduction level of PDMA-Fe was greater than that of EDTA-Fe and citrate-Fe under alkaline $\mathrm{pH}$.

Conclusions PDMA-Fe is utilized by cucumber roots more efficiently than traditional synthetic chelates in both calcareous substrate and hydroponic cultures. The higher availability of PDMA-Fe may be attributed to its higher reducibility. Our findings suggest that PDMA-Fe could be a good Fe fertilizer for dicots.

Keywords Mugineic acid - Iron deficiency · Chlorosis $\cdot$ Chelate $\cdot$ Reduction $\cdot$ EDDHA

\section{Introduction}

Iron $(\mathrm{Fe})$ is the fourth most abundant element in the Earth's crust; however, under aerobic conditions, the concentrations of $\mathrm{Fe}^{3+}$ and $\mathrm{Fe}^{2+}$ ions in soil solution are below $10^{-15} \mathrm{M}$ at $\mathrm{pH}>6$, thus limiting plant growth at neutral or alkaline $\mathrm{pH}$ (Lindsay and Schwab 1982; Guerinot and Yi 1994). Therefore, plants have developed two mechanisms for Fe acquisition (called "Strategy I" and "Strategy II") to cope with this limitation (Marschner and Römheld 1986). Strategy II plants, which are mostly grasses, respond to Fe deficiency by secreting phytosiderophores (PS) into the rhizosphere which solubilize Fe(III) (Takagi 1976; Ma and Nomoto 1996; Ueno et al. 2007; Nozoye et al. 2011). Plants then take up PS-Fe complexes through yellow stripe 1-like (YSL) transporters without prior reduction (Curie et al. 2001; Murata et al. 2006; Inoue et al. 2009). In contrast, Strategy I plants, including non-grass monocots and dicots, release organic compounds including flavins and iron-mobilizing coumarins in response to $\mathrm{Fe}$ deficiency to mobilize rhizosphere $\mathrm{Fe}$ through reduction and/or chelation (Römheld and Marschner 1983; Jin et al. 2007; Sisó-Terraza et al. 2016; Tsai and Schmidt 2017). Iron chelates are then reduced by a plasma membrane-bound $\mathrm{Fe}(\mathrm{III})$ reductase and subsequently taken up via $\mathrm{Fe}^{2+}$ transporters, such as iron-regulated transporter 1 (IRT1) (Eide et al. 1996). The acidification of the rhizosphere by $\mathrm{H}^{+}$release is also required to increase $\mathrm{Fe}$ solubility and to ensure $\mathrm{Fe}$ (III) reductase activity in this strategy (Römheld and Nikolic 2007). Based on the difference in mechanisms, sparingly soluble $\mathrm{Fe}$ (III) is considered a substrate for the Strategy II system, whereas Fe(III) chelates are the major Fe sources for the Strategy I system (Römheld and Marschner 1986).

To correct Fe deficiency in crops that insufficiently induce adaptive responses to Fe starvation, synthetic $\mathrm{Fe}$ chelates such as Fe-ethylenediamine- $N, N, N^{\prime}, N^{\prime}-$ tetraacetic acid complex (EDTA-Fe) and Fe-ethylenediamine- $N, N$ '-bis(2-hydroxy-phenyl acetic acid) complex (EDDHA-Fe) are supplied to the soil as Fe fertilizers (Lucena 2006; Römheld and Nikolic 2007). Among them, EDDHA-Fe is the most effective fertilizer for increasing soluble $\mathrm{Fe}$ in calcareous soil because of its higher stability constant at high pH (López-Rayo et al. 2009). However, the non-biodegradability of these chelates and their accumulation in the environment remains a concern (Nowack 2002; Hyvönen et al. 2003; Schenkeveld et al. 2012). Several biodegradable chelates have been studied as alternatives to traditional chelates (Pinto et al. 2014). Some of them were demonstrated to have a similar or high phytoavailability compared with EDTA-Fe for various plant species when applied to hydroponic or soilless culture media (Villén et al. 2007; Nowack et al. 2008; Hasegawa et al. 2011, 2012). However, none of them had greater efficacy than EDDHA-Fe under both culture conditions (Albano and Merhaut 2012; López-Rayo et al. 2019). Application of microbial siderophores to roots has been proposed as an environmentally friendly alternative to provide Fe to plants (Vansuyt et al. 2007; Nagata et al. 2013; Ahmed and Holmström 2014). However, the redox potential of $\mathrm{Fe}$ (III) captured by microbial siderophores is generally too low to be reduced by the roots. Thus, microbial siderophore-Fe has a low availability to plants unless high reducibility is imparted (Ueno et al. 2019) or the ligand is substituted with PS (Ahmed and Holmström 2014).

Recently, a novel synthetic PS, proline-2'deoxymugineic acid (PDMA), has been developed as a promising Fe fertilizer (Suzuki et al. 2021). The key structural difference between PDMA and natural 2'-deoxymugineic acid (DMA) is the substitution of L-proline for L-azetidine, which contributes to moderate biodegradability and decrease synthesis cost. Application of PDMA with or without Fe to calcareous soil promoted $\mathrm{Fe}$ uptake in rice plants more effectively than EDDHA-Fe and EDTA-Fe. The higher availability of PDMA-Fe was attributed to the fact that PDMA-Fe can be directly taken up by YSL transporters. It has been suggested that dicot plants can 
utilize PS when intercropped with grasses (Zuo et al. 2000; Ma et al. 2003; Cesco et al. 2006; Ueno and Ma 2009). Furthermore, recent studies have implied the secretion of PS from dicots, such as tomato (Astolfi et al. 2020) and grapevine (Marastoni et al. 2020). Therefore, in the present study, we aimed to evaluate the availability of PDMA-Fe to the Strategy I system using dicots. We investigated the effects of PDMAFe application on a calcareous substrate and the availability depending on $\mathrm{pH}$ in hydroponic cultures, and revealed that PDMA-Fe could be an alternative to the traditional synthetic chelates, even for Strategy I plants.

\section{Materials and methods}

Preparation of $\mathrm{Fe}(\mathrm{III})$ chelates

The chemical synthesis of PDMA has been described previously (Suzuki et al. 2021). To prepare the Fe(III) complex, $2.47 \mathrm{M} \mathrm{FeCl}_{3}$ and $10 \mathrm{mM}$ PDMA were mixed at a molar ratio of $2: 1$. The $\mathrm{pH}$ of the solution was adjusted to 7.0 with $0.5 \mathrm{M} \mathrm{NaOH}$ to precipitate the excess $\mathrm{Fe}^{3+}$ as $\mathrm{Fe}$ (III) oxide-hydroxides or $\mathrm{Fe}(\mathrm{III})$ oxides. The suspension was then incubated at $50{ }^{\circ} \mathrm{C}$ for $1 \mathrm{~h}$ with occasional mixing and then centrifuged at $15,000 \mathrm{rpm}$ for $3 \mathrm{~min}$. The supernatant containing PDMA-Fe was passed through a $0.22-\mu \mathrm{m}$ syringe filter (Hawach Scientific, Xi'an, China) to further exclude precipitated $\mathrm{Fe}$. Fe(III) citrate (Cit-Fe) was prepared similarly using citric acid (Cas No. 5949-291; Wako, Tokyo, Japan) instead of PDMA. Both Fe chelates were stored at $-30{ }^{\circ} \mathrm{C}$ until use to avoid biodegradation. EDDHA-Fe (Dissolvine Q-Fe-6; Akzo Nobel, Amsterdam, the Netherlands) and EDTA-Fe (Cas No. 15708-41-5; Dojindo Laboratories, Kumamoto, Japan) were also used in the experiments.

\section{Plant material and culture condition}

The availability of PDMA-Fe to Strategy I plants was tested using cucumber (Cucumis sativus L., 'Hokushin'; Takii, Kyoto, Japan). For the calcareous substrate culture, the seeds were germinated in moistened vermiculite at $27{ }^{\circ} \mathrm{C}$ for $4 \mathrm{~d}$. After germination, the seedlings were transferred to pots (one plant per pot), $4.5-6.0 \mathrm{~cm}$ in diameter and $5 \mathrm{~cm}$ tall, filled with $100 \mathrm{~g}$ calcareous substrate consisting of shelly fossils $\left[\mathrm{pH}\left(\mathrm{H}_{2} \mathrm{O}\right) 9.1,10-15 \mathrm{~g} \mathrm{Fe} \mathrm{kg}^{-1}\right.$ substrate dry weight] purchased from Nihonkai Hiryo Co., Ltd., Takaoka, Japan. The calcareous substrate was mined from a site analyzed previously (Morikawa et al. 2004). The substrate was fertilized with N-P-K fertilizer (15-15-10; Chiyodakasei, SunAgro, Toyama, Japan) at $3 \mathrm{~g} \mathrm{~kg}^{-1}$ substrate dry weight and watered daily with distilled water to the saturation level $(35 \mathrm{~mL} / 100 \mathrm{~g}$ substrate dry weight/pot). Seedlings were grown for $10 \mathrm{~d}$ until true leaves expanded. The seedlings with true leaves showing chlorosis were selected, and the substrate was supplemented with or without PDMA-Fe, EDDHA-Fe, or Cit-Fe. The concentration of each Fe chelate in $35 \mathrm{~mL}$ substrate solution was adjusted to $30 \mu \mathrm{M}(0.586 \mathrm{mg} \mathrm{Fe}$ $\mathrm{kg}^{-1}$ substrate dry weight). Plants were grown in a controlled growth chamber [ $14 \mathrm{~h}$ of light at $27{ }^{\circ} \mathrm{C}$ / $10 \mathrm{~h}$ of dark at $22{ }^{\circ} \mathrm{C}$; light intensity (metal halide lamp) $75-100 \mu \mathrm{mol}$ photons $\mathrm{m}^{-2} \mathrm{~s}^{-1}$; relative humidity $50-60 \%$ ] for $4 \mathrm{~d}$. SPAD values in expanded true leaves were analyzed daily using a chlorophyll meter (SPAD-502Plus, Konica Minolta, Tokyo, Japan). The experiment was also performed on pumpkin (Cucurbita moschata L. 'YūYūikki' white type; Saitama Gensyu Ikuseikai, Kuki, Japan).

For the hydroponic culture, seeds were germinated on moistened filter paper in Petri dishes at $25-27{ }^{\circ} \mathrm{C}$ for $1-2 \mathrm{~d}$ in the dark. Germinated seedlings were transferred to a net floated on $0.5 \mathrm{mM} \mathrm{CaCl} 2$ and incubated for 2-3 d. Subsequent culture was carried out under the same controlled condition described above. Seedlings were transferred to $1.25 \mathrm{~L}$ pots (four plants per pot) and pre-cultured with $1 / 5$ Hoagland nutrient solution ( $\mathrm{pH}$ 5.8) containing the following macroelements (in $\mathrm{mM}$ ): $\mathrm{KNO}_{3}(1), \mathrm{Ca}\left(\mathrm{NO}_{3}\right)_{2}$ (1), $\mathrm{MgSO}_{4}(0.4)$, and $\left(\mathrm{NH}_{4}\right) \mathrm{H}_{2} \mathrm{PO}_{4}(0.2)$, and microelements (in $\mu \mathrm{M}$ ): $\mathrm{H}_{3} \mathrm{BO}_{3}$ (3), $\mathrm{MnCl}_{2}(0.5), \mathrm{ZnSO}_{4}$ (0.4), $\mathrm{CuSO}_{4}(0.2)$, and $\left(\mathrm{NH}_{4}\right)_{6} \mathrm{Mo}_{7} \mathrm{O}_{24}(0.2)$. Fe was not added to the nutrient solution to induce Fe-deficiency chlorosis. To investigate the effect of PDMA$\mathrm{Fe}$ on relieving Fe deficiency, seedlings grown for a week were exposed to a treatment solution containing macroelements and 0.5 $\mu \mathrm{M}$ PDMA-Fe, EDDHA$\mathrm{Fe}$, or Cit-Fe. The treatment solution was buffered with $1 \mathrm{mM}$ piperazine-1,4-bis(2-ethanesulfonic acid) (PIPES)-NaOH (pH 7.0), 3-[4-(2-hydroxyethyl)1-piperazinyl]propanesulfonic acid (EPPS)-NaOH 
(pH 8.0), or $N$-cyclohexyl-2-aminoethanesulfonic acid (CHES)-NaOH (pH 9.0). The solution was continuously aerated and replenished daily, and the $\mathrm{pH}$ was adjusted twice per day. The SPAD value in expanded true leaves was recorded daily during the 4 $\mathrm{d}$ of treatment. The availability of PDMA-Fe was also compared with that of a synthetic chelate with high stability in the high $\mathrm{pH}$ range (7.5-12), $N, N^{\prime}$-bis(2hydroxybenzyl)ethylenediamine- $N, N$ '-diacetic acid (HBED)-Fe(III) (Adob, Poznan, Poland) (Ma et al. 1994); a natural microbial siderophore, deferoxamine B (DFOB)-Fe(III) (Sigma-Aldrich); and a synthetic microbial siderophore with high reducibility, tris[2$\{(N$-acetyl- $N$-hydroxy $)$ glycylamino $\}$ ethyl $]$ amine (TAGE)-Fe(III) (Matsumoto et al. 2001; Ueno et al. 2019) at $\mathrm{pH} 8.0$.

After the treatments, plants were divided into roots, true leaves, and the other aerial parts (cotyledons and stems), washed twice with distilled water, blotted, and used for elemental concentration measurements.

\section{Determination of elemental concentrations}

Harvested samples were dried at $70{ }^{\circ} \mathrm{C}$, weighed, digested with $60 \%(\mathrm{v} / \mathrm{v}) \mathrm{HNO}_{3}$ at $140{ }^{\circ} \mathrm{C}$, and then diluted with distilled water to appropriate concentrations. The concentrations of $\mathrm{Fe}, \mathrm{Zn}, \mathrm{Mn}$, and $\mathrm{Cu}$ in the digested solution were determined using atomic absorption spectrometry (AA-6800, Shimadzu, Kyoto, Japan).

\section{Quantitative RT-PCR}

Expression of $\mathrm{Fe}$ deficiency-responsive genes, ferric reduction oxidase 1 (CsFRO1) (Acc. No. AY590765.1, https://www.ncbi.nlm.nih.gov/) and iron-regulated transporter 1 (CsIRTI) (Acc. No. XM_004145406.3), were compared among Fe chelate treatments to estimate differences in availability. Seedlings were pre-cultured in the absence of Fe for $4 \mathrm{~d}$, and then exposed to a nutrient solution (pH 9.0, $1 \mathrm{mM}$ CHES-NaOH) containing $0.5 \mu \mathrm{M}$ PDMA-Fe, EDDHA-Fe, or Cit-Fe for $72 \mathrm{~h}$. The $\mathrm{pH}$ of the nutrient solution was adjusted three times per day. The nutrient solution was replenished $48 \mathrm{~h}$ after the Fe treatment. The total RNA was extracted from the roots using the ISOSPIN Plant RNA kit (Nippon Gene, Tokyo, Japan) at $4 \mathrm{~h}$ after lighting, treated with DNase I (Toyobo, Osaka, Japan), and converted to
cDNA using ReverTra Ace (Toyobo). Gene expression was measured using quantitative reverse transcription polymerase chain reaction (qRT-PCR) with the primers 5'-TGTGGGCAACAACTATTCCTC$3^{\prime}$ and $5^{\prime}$-AGGAGATGCCAACATGGAAG-3' for CsFRO1, and 5'-CTCATTGCGAGTGTCATTGG-3' and 5'-GAATGATACCTGCTGCGAAAG-3' for CsIRT1. Actin 7 (Acc. No. XM_011659465.2), used as an internal control, was analyzed using the primers 5'-TTGCAGACAGGATGAGCAAG-3' and 5'-ACC CTCCAATCCAAACACTG-3'. qRT-PCR was carried out using a KOD SYBR qPCR mix (Toyobo) on a Prism 7300 Real Time PCR System (Applied Biosystems, Foster City, CA, USA).

Reducibility assay

The reducibility of $\mathrm{Fe}(\mathrm{III})$ chelates by plant roots was analyzed according to Romera et al. (1996), with some modifications. Roots (approx. $0.15 \mathrm{~g}$ fresh weight) of intact cucumber grown without $\mathrm{Fe}$ for 5 $\mathrm{d}$ were exposed to $10 \mathrm{~mL}$ of assay solution $(0.2 \mathrm{mM}$ $\mathrm{CaSO}_{4}, 5 \mathrm{mM}$ PIPES at pH 7.0, EPPS at $\mathrm{pH}$ 8.0, or CHES at $\mathrm{pH}$ 9.0), supplemented with an Fe source (0.1 mM PDMA-Fe, EDTA-Fe, or Cit-Fe) and 0.2 $\mathrm{mM}$ bathophenanthroline disulfonic acid [BPDS] [Cas. No. 98645-86-4, Dojindo Laboratories]), for $1 \mathrm{~h}$, with occasional mixing at $25{ }^{\circ} \mathrm{C}$ in the dark. The absorbance of the solution was read at $535 \mathrm{~nm}$ using a spectrophotometer (V-630Bio, Jasco, Tokyo, Japan). After subtracting the A535 of the solution without the plant from that of the respective solution, the BPDS-Fe(II) concentration was calculated using an extinction coefficient of $22.14 \mathrm{mM}^{-1} \mathrm{~cm}^{-1}$. The fresh weight of the roots was also recorded to calculate the reduction rates. In this assay, both plasma membrane bound reductase and reducing-compound exudations mediated the reduction.

Measurement of $\mathrm{O}_{2}$-evolution rate

$\mathrm{O}_{2}$ exchange was monitored using a ROS Field Master (RFM) with a closed leaf-type chamber (Bunkoukeiki Co., Ltd, Tokyo, Japan). An RFM is a device that can simultaneously measure P700 absorption and determine the oxygen evolution rate. The device consists of a measurement light, farred light, actinic red light, LED light source unit, closed chamber (including light detector, oxygen 
measurement sensor, and temperature/humidity/ pressure sensor), signal processing unit, and touch panel display as the user interface. The device is powered by a $12 \mathrm{~V}$ lithium-ion battery. The sample was irradiated with a $16 \mathrm{~mm} \times 16 \mathrm{~mm}$ light spot from the light guide path, and the transmitted light was received by a photodetector. In addition, the oxygen concentration in the closed chamber was measured using a galvanic oxygen sensor. The conversion of the sensor signal for oxygen measurement was calculated from the oxygen concentration in $1 \mathrm{~mL}$ of air and the amount of signal change, and the oxygen change was proportional to the measurement signal. In addition, the temperature, humidity, and atmospheric pressure inside the chamber were measured to compensate for the signal value of the oxygen sensor. The closed chamber has two doorways, one of which can be fitted with a tube to allow human exhalation to saturate the interior of the chamber to a saturated $\mathrm{CO}_{2}$ state. As a result, the inside of the closed chamber can be brought into a saturated $\mathrm{CO}_{2}$ state, and the maximum photosynthetic activity can be measured. A leaf disc $\left(2.5 \mathrm{~cm}^{2}\right)$ excised from the true leaves of seedlings cultured with Fe chelate (PDMA-Fe, EDDHA-Fe, or Cit-Fe) at $\mathrm{pH} 9.0$ for $6 \mathrm{~d}$ was placed in the chamber. Actinic red light $(660 \mathrm{~nm})$ was illuminated from the top of the chamber, and the photon flux density (PFD) was adjusted to $1000 \mu \mathrm{mol}$ photons $\mathrm{m}^{-2} \mathrm{~s}^{-1}$. As the chamber is a closed system, $\mathrm{CO}_{2}$ in the chamber was consumed during photosynthesis; therefore, additional $\mathrm{CO}_{2}$ was supplied with expiratory air (assumed to be $\mathrm{CO}_{2}$ saturated air).

Statistical analysis

Data were analyzed using Tukey's tests with BellCurve for Excel (Social Survey Research Information, Tokyo, Japan). Significant differences $(P<0.01$ or $P$ $<0.05)$ are indicated by different letters.

\section{Results}

Effects of PDMA-Fe application to a calcareous substrate

To evaluate PDMA-Fe as an Fe source for Strategy I plants, we used cucumber and examined the effect of $30 \mu \mathrm{M}$ PDMA-Fe application to the substrate using other Fe chelates as controls. At $4 \mathrm{~d}$ after the treatment, plants treated with $\mathrm{Cit}-\mathrm{Fe}$ and with no $\mathrm{Fe}$ (control) showed Fe-deficiency chlorosis, whereas plants treated with PDMA-Fe and EDDHA-Fe did not (Fig. 1a). The SPAD values in the Cit-Fe and control plants were similar, ranging from 17 to 21 during the treatment (Fig. 1b). In contrast, the SPAD value
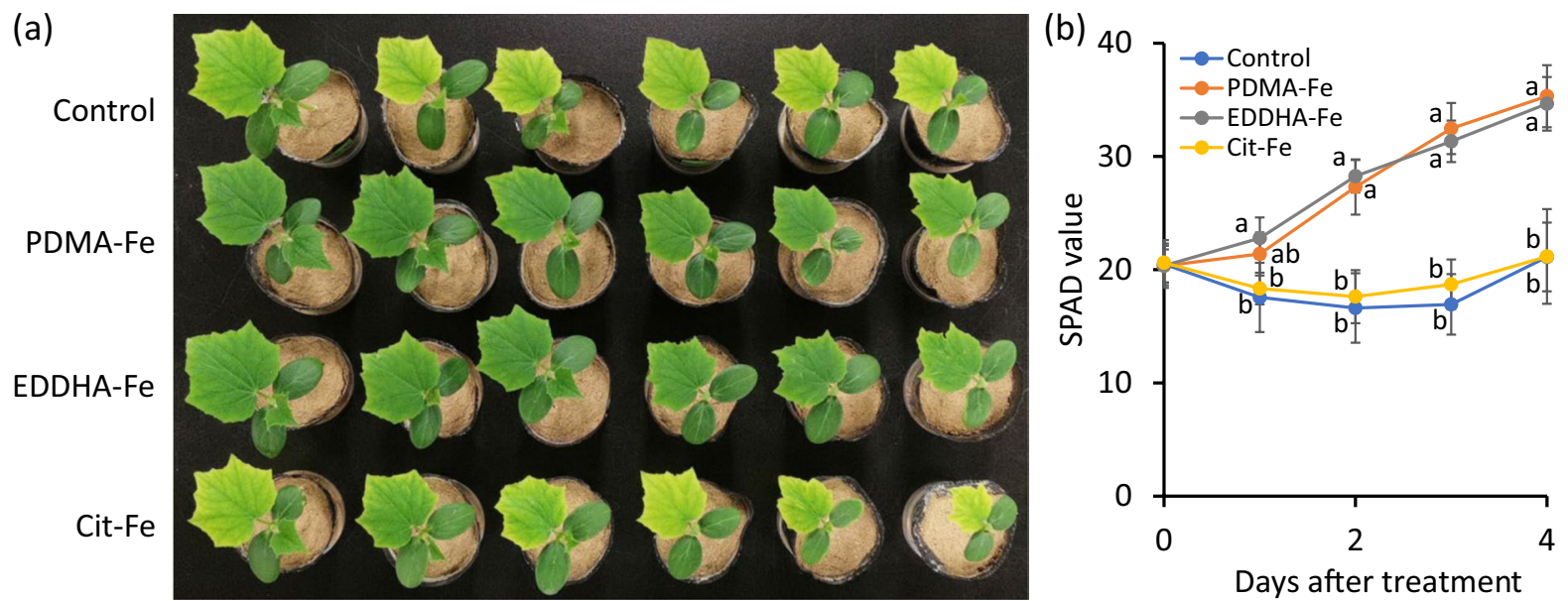

Fig. 1 Effects of PDMA-Fe(III) on leaf chlorophyll in cucumber grown in a calcareous substrate. Iron-deficient seedlings were treated with or without Fe chelates. (a) Shoot image on day 4 and (b) time course of chlorophyll level (SPAD value) in expanded true leaves. Data are presented as mean \pm standard deviation $(n=6)$. Different letters indicate significant differences $(P<0.01)$ using Tukey's test 
increased progressively from 20 to 35 in the PDMAFe and EDDHA-Fe treatments (Fig. 1b).

For elemental analysis, the shoots were divided into true leaves and other parts, including cotyledons and stems. Iron accumulated in the true leaves is likely to be derived from the soil solution containing $\mathrm{Fe}$ chelates, because $\mathrm{Fe}$ is rarely re-translocated from older leaves to younger leaves (Römheld and Nikolic 2007), whereas Fe accumulated in the other parts originate from both seed and substrate solutions. Thus, the true leaves can provide an estimate of the rate of Fe uptake from $\mathrm{Fe}$ chelates in comparison with the whole shoot. The Fe concentration in the true leaves in the PDMA-Fe treatment was $34 \%$ higher than that in the EDDHA-Fe treatment, and more than twice as high as that in the Cit-Fe treatment and control (Fig. 2a). The Fe concentration in the other aerial parts was also significantly higher in the PDMA-Fe treatment than in the other treatments (differences significant at $P<0.05$ ), but to a less degree (Fig. 2a). A greater effect of PDMA-Fe than EDDHA-Fe was also observed in pumpkin (Supplementary Fig. S1). In the true leaves, the $\mathrm{Zn}$ concentration was more than 1.5-fold higher than that in the other treatments (Fig. 2b), whereas the Mn concentration was almost half of that in the control and Cit-Fe treatment, it but did not differ from that in the EDDHA-Fe treatment (Fig. 2c). In cotyledons and stems, both $\mathrm{Zn}$ and $\mathrm{Mn}$ concentrations showed similar tendencies to those in the true leaves but to a less extent (Fig. 2b, c). The $\mathrm{Cu}$ concentration did not differ significantly among the treatments (Fig. 2d). The higher shoot Fe concentration under PDMA-Fe application supports that PDMA-Fe is more available than the other Fe chelates in alkaline substrates.

Efficacy of PDMA-Fe to relieve Fe deficiency in hydroponics at various $\mathrm{pH}$ levels

To investigate the pH-dependent efficacy of PDMA$\mathrm{Fe}$ to improve $\mathrm{Fe}$ chlorosis, we applied Fe chelates in hydroponics under neutral-alkaline $\mathrm{pH}$. After $4 \mathrm{~d}$
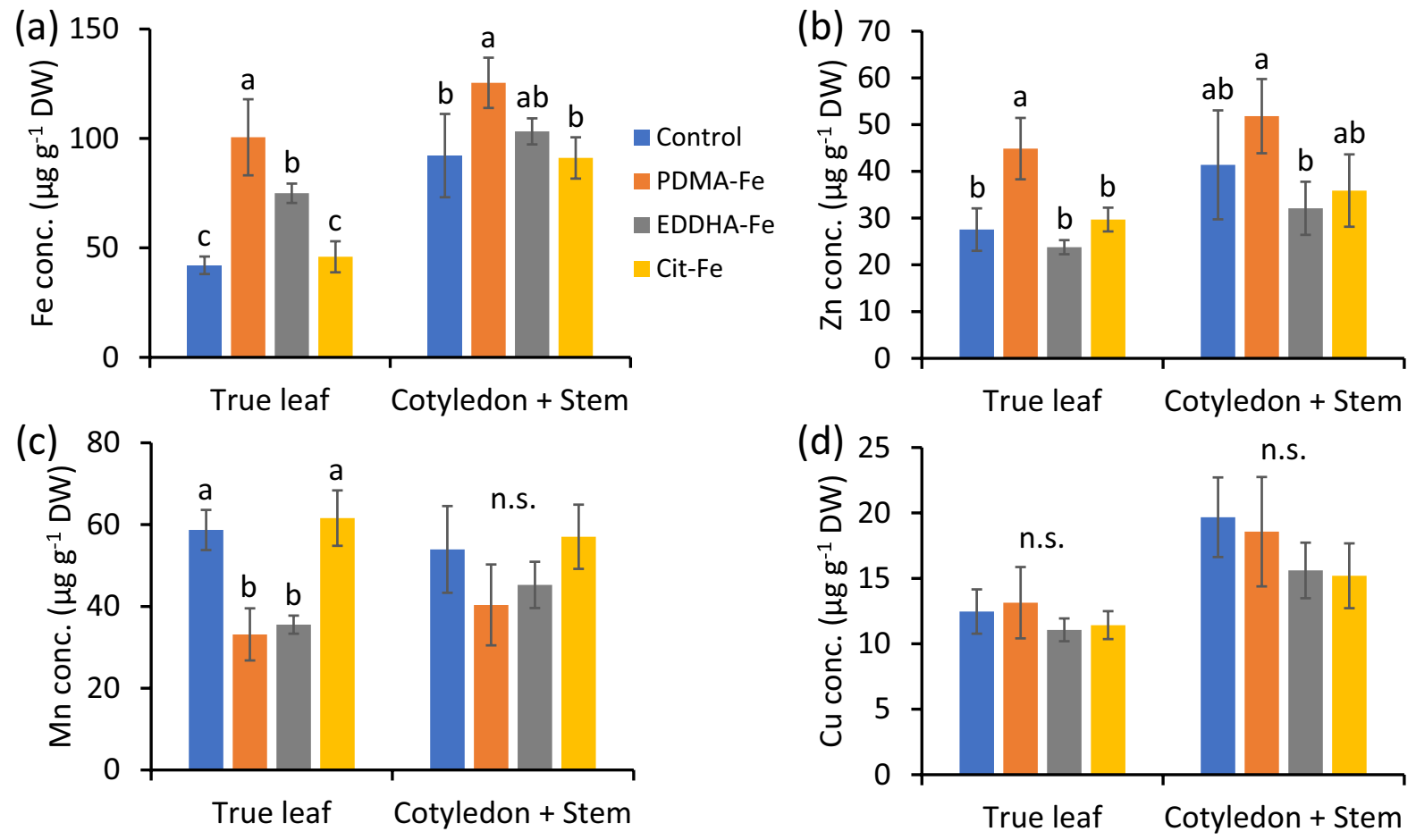

Fig. 2 Effects of PDMA-Fe(III) on microelement concentrations in cucumber grown in a calcareous substrate. Seedlings were treated with or without Fe chelates for $4 \mathrm{~d}$. Concentrations of $\mathrm{Fe}(\mathbf{a}), \mathrm{Zn}(\mathbf{b}), \mathrm{Mn}(\mathbf{c})$, and $\mathrm{Cu}(\mathbf{d})$ in true leaves and

other aerial parts (cotyledons and stems). Data are presented as mean \pm standard deviation $(n=6)$. Different letters indicate significant differences $(P<0.01)$ using Tukey's test. n.s., not significant 

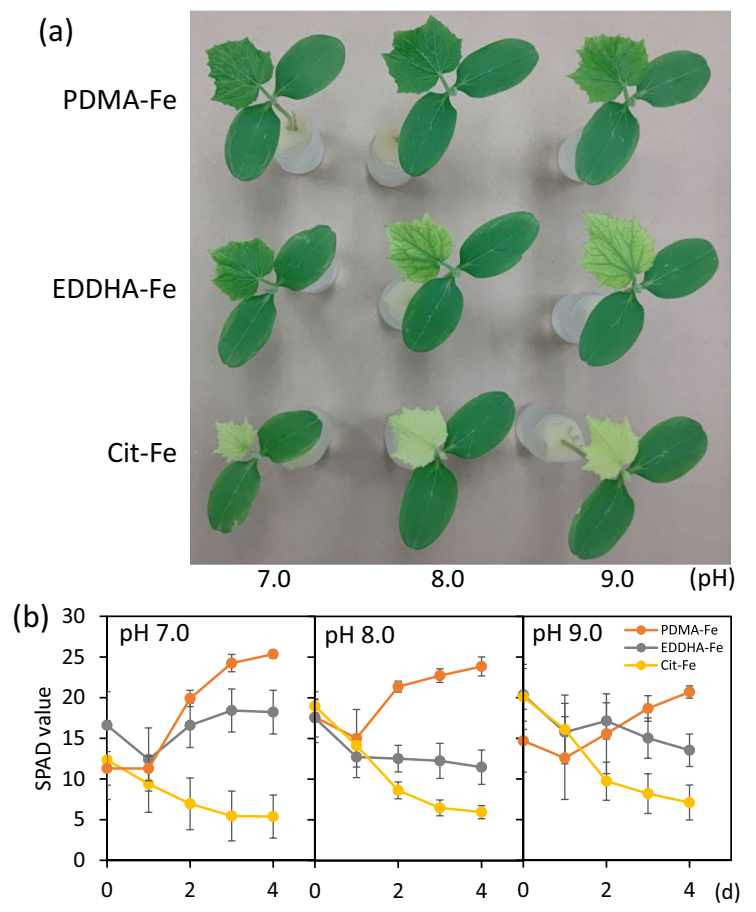

Fig. 3 Effects of PDMA-Fe(III) on improving Fe chlorosis in hydroponic culture. Seedlings were pre-cultured in the absence of $\mathrm{Fe}$ for a week and were treated with $0.5 \mu \mathrm{M}$ Fe chelates under neutral-alkaline $\mathrm{pH}(7.0,8.0$, and 9.0) for $4 \mathrm{~d}$. (a) Representative images of shoots on day 4 and (b) time course chlorophyll level (SPAD value) in expanded true leaves. Data are presented as mean \pm standard deviation $(n=4)$

of exposure, PDMA-Fe relieved Fe chlorosis more effectively than EDDHA-Fe at all pH levels (Fig. 3a). The SPAD value increased with time in the PDMAFe treatment and exhibited 1.4-, 2.1-, and 1.5-fold higher rates at $\mathrm{pH} 7.0,8.0$, and 9.0, respectively, than that under the EDDHA-Fe treatment on day 4 $(P<0.01$; Fig. 3b). In the Cit-Fe treatment, Fe-deficient chlorosis did not improve regardless of $\mathrm{pH}$, and the SPAD value decreased with time and reached significantly lower levels than that in the EDDHA-Fe treatment on day $4(P<0.01$; Fig. 3a, b). Recovery from Fe deficiency was also determined by analyzing the rate of $\mathrm{O}_{2}$-evolution as an index of PSII activity in true leaves of cucumber grown at $\mathrm{pH}$ 9.0. The rate of $\mathrm{O}_{2}$-evolution increased and reached a steady state at about $3 \mathrm{~min}$ in the PDMA-Fe and EDDHAFe treatments, whereas a steady negative rate indicating respiration was observed in the Cit-Fe treatment (Supplementary Fig. S2). The rate in the PDMA-Fe treatment was twice as high as that in the EDDHA-Fe
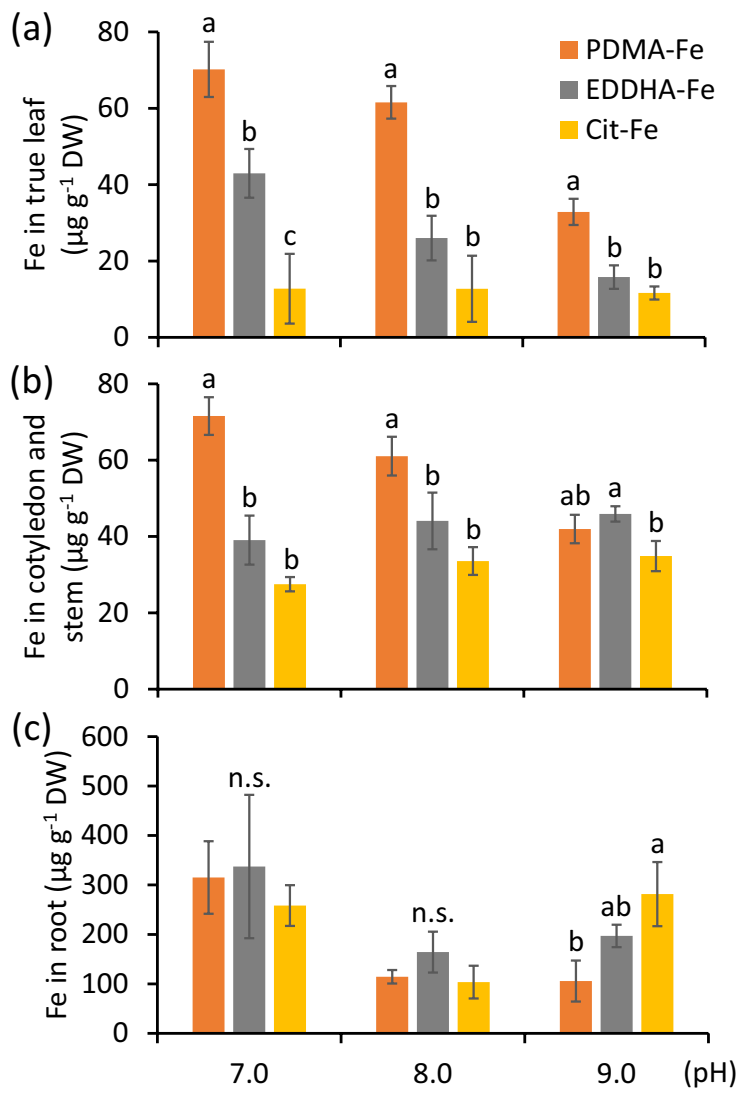

Fig. 4 Effect of PDMA-Fe(III) on Fe accumulation in hydroponic culture. Seedlings pre-cultured in the absence of Fe for a week were treated with $0.5 \mu \mathrm{M}$ Fe chelates under neutralalkaline $\mathrm{pH}(7.0,8.0$, and 9.0) for $4 \mathrm{~d}$. Concentration of $\mathrm{Fe}$ in the true leaves (a), cotyledons and stems (b), and roots (c). Data are presented as mean \pm standard deviation $(n=4)$. Different letters indicate significant differences $(P<0.01)$ using Tukey's test. n.s., not significant

treatment under the steady state $(P<0.01$; Supplementary Fig. S2).

The Fe concentration in the true leaves was 1.6and 5.5-fold higher under the PDMA-Fe treatment than under the EDDHA-Fe and Cit-Fe treatments at $\mathrm{pH}$ 7.0, respectively (Fig. 4a). The concentration in the PDMA-Fe treatment decreased with elevated $\mathrm{pH}$, but it was still more than twice as high as that in the other treatments (Fig. 4a). In the cotyledons and stems, the Fe concentration was also significantly higher under the PDMA-Fe treatment than under the other treatments at $\mathrm{pH} 7.0$ and 8.0, but it did not differ at $\mathrm{pH} 9.0$ (Fig. 4b). In the roots, the Fe concentration did not differ among the treatments at $\mathrm{pH} 7.0$ or 8.0 , 
but it was higher in the Cit-Fe treatment than in the PDMA-Fe treatment at pH 9.0 (Fig. 4c).

The comparative analysis of Fe chelates showed that the effect on the chlorophyll level decreased in the following order: PDMA-Fe $>$ EDDHA-Fe and TAGE-Fe > EDTA-Fe, HBED-Fe, Cit-Fe, DFOB-Fe, and $-\mathrm{Fe}$ (Supplementary Fig. S3a), whereas that on shoot $\mathrm{Fe}$ concentration was in the following order: PDMA-Fe $>$ EDDHA-Fe and TAGE-Fe $>$ EDTA$\mathrm{Fe}$, HBED-Fe and Cit-Fe $>$ DFOB-Fe and $-\mathrm{Fe}$ (differences significant at $P<0.05$; Supplementary Fig. S3b).

Effects of PDMA-Fe on expression of $\mathrm{Fe}$ deficiency-inducible genes

The higher availability of PDMA-Fe at various $\mathrm{pH}$ levels was further verified by analyzing the expression of two Fe deficiency-inducible genes, CsFROI and CSIRT1, which have been demonstrated to be involved in ferric reduction and ferrous uptake in cucumber roots, respectively (Waters et al. 2007). At $72 \mathrm{~h}$ after $\mathrm{Fe}$ chelate application to Fe-deficient plants, CsFRO1 expression in the PDMA-Fe treatment was approximately two-fifths of that in the EDDHA-Fe treatment (differences significant at $P<0.05)$ and one-fifth of that in the Cit-Fe treatment (Fig. 5a). CsIRT1 expression under the PDMA-Fe treatment was similar to that under the EDDHA-Fe treatment, but $41 \%$ lower than that under the Cit-Fe treatment (Fig. 5b).

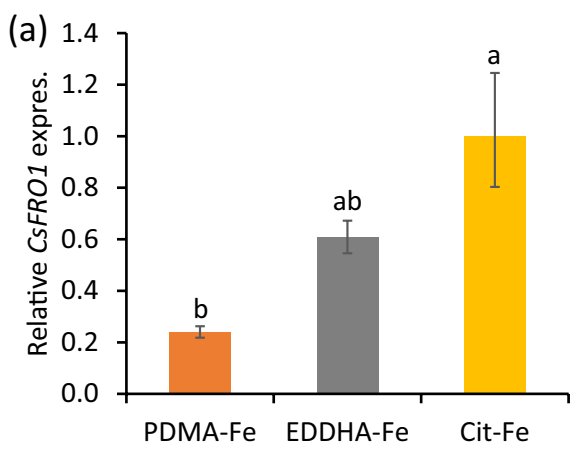

Fig. 5 Effects of PDMA-Fe(III) on Fe status. Seedlings precultured in the absence of $\mathrm{Fe}$ for $4 \mathrm{~d}$ were exposed to a nutrient solution ( $\mathrm{pH}$ 9.0) containing $0.5 \mu \mathrm{M}$ Fe chelates for $72 \mathrm{~h}$. Relative expression levels of ferric reduction oxidase 1 (CsFRO1) (a) and iron-regulated transporter 1 (CsIRT1) (b) in the roots
Reducibility of PDMA-Fe

To determine the mechanism underlying the higher availability of PDMA-Fe, we assayed the reducibility of Fe from $\mathrm{Fe}$ chelates by cucumber roots. Colorless EDTA-Fe was used instead of EDDHA-Fe because EDDHA-Fe has a strong absorption band in the near region as BPDS-Fe(II) forms in this assay. The rate of PDMA-Fe reduction was $34 \%$ higher than that of EDTA-Fe at pH 7.0 (Fig. 6). The difference in reducibility increased with $\mathrm{pH}$; the rates were 3.4 - and 5.6-times higher at $\mathrm{pH} 8.0$ and 9.0, respectively, in

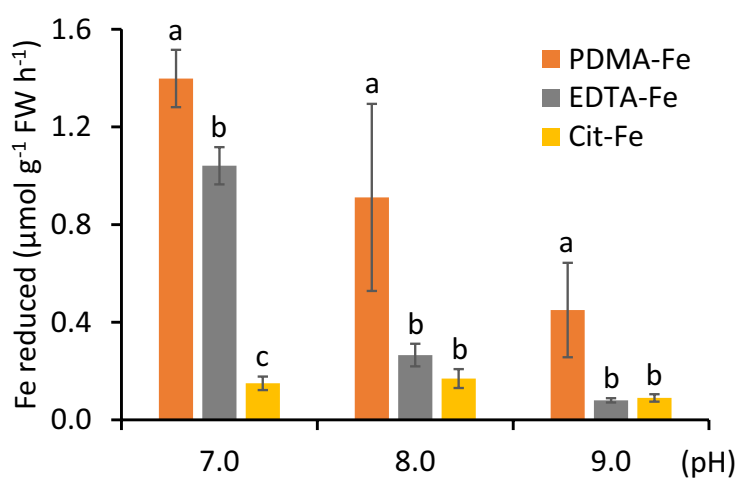

Fig. 6 Reducibility of $\mathrm{Fe}(\mathrm{III})$ from Fe chelates by cucumber roots. Roots of cucumber plants grown hydroponically without Fe for $5 \mathrm{~d}$ were used. Reducibility was examined under neutral-alkaline $\mathrm{pH}(7.0,8.0$, and 9.0) using the bathophenanthroline disulfonic acid assay. Data are presented as mean \pm standard deviation $(n=3)$. Different letters indicate significant differences $(P<0.05)$ using Tukey's test

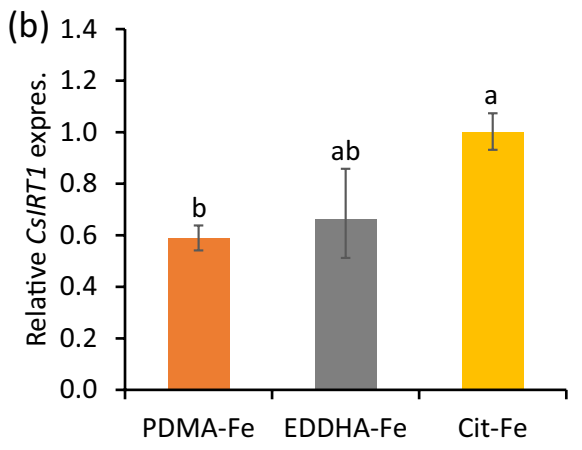

grown under the Cit-Fe(III) treatment. Actin 7 was used as the internal control. Data are presented as mean \pm standard deviation $(n=3)$. Different letters indicate significant differences $(P<0.01)$ using Tukey's test 
PDMA-Fe than in EDTA-Fe. The reducibility of CitFe was significantly lower than that of PDMA-Fe and EDTA-Fe at $\mathrm{pH} 7.0$ and 8.0 but was similar to that of EDTA-Fe at $\mathrm{pH} 9.0$.

\section{Discussion}

Grass-borne PS and microbial siderophores can solubilize Fe in the soil (Takagi et al. 1988; Ahmed and Holmström 2014), and the resultant Fe complex can be utilized by dicots as substrates of the reductionbased Fe acquisition system (Römheld and Marschner 1986). In the first study of synthetic PDMA (Suzuki et al. 2021), it was demonstrated that the PDMA-Fe complex can be directly taken up by grasses, leading to a higher availability than traditional chelates to the Strategy II system. Here, we provide evidence that PDMA-Fe could also be a good Fe source for Strategy I plants.

In the present study, PDMA-Fe provided more Fe than the other chelates in calcareous substrate and hydroponic culture (Figs. 2 and 4). Consistent with Fe accumulation, the chlorophyll level and PSII activity were recovered substantially by PDMA-Fe application (Figs. 1 and 3, Supplementary Fig. S2) because $\mathrm{Fe}$ is needed for many chloroplast components (Broadley et al. 2012). The application of PDMAFe lowered the expression of Fe deficiency-inducible genes more than the other Fe chelates (Fig. 5), suggesting that the Fe status was more effectively improved. Although the constant stability of PDMA to $\mathrm{Fe}(\mathrm{III})(\log K=17.1)$ was lower than that of EDTA ( $\log K=25.1)$, EDDHA $(o, o$-EDDHA [ $\log K=35.1]$, and $o, p$-EDDHA [ $\log K=28.7])$, the higher Fe uptake from PDMA-Fe may be attributed to higher reducibility (Fig. 6). In the case of EDDHA-Fe, its high availability is due to the formation of highly reducible species, which are induced by the lowering of $\mathrm{pH}$ by root $\mathrm{H}^{+}$release (Gómez-Gallego et al. 2005). Therefore, decreased Fe uptake with increasing pH (Fig. 4) may be due to decreased formation of such species as well as to the inhibition of reductase activity (Römheld and Marschner 1986). Although the chemical mechanism underlying the higher reducibility of PDMA$\mathrm{Fe}$ requires clarification, the reduction of PDMA-Fe could be structurally less sensitive to high $\mathrm{pH}$ in comparison with that of traditional synthetic Fe chelates.
Thus, PDMA-Fe seems to be effective for crops relying on the reduction-based $\mathrm{Fe}$ uptake system.

Another possible explanation for the high availability of PDMA-Fe is that a portion of this complex may be directly taken up by roots via YSL transporters. There is evidence that Strategy I plants can directly take up PS-Fe in intercropping systems. Xiong et al. (2013) reported that DMA secreted by intercropped maize was detected in peanut (Arachis hypogaea L.) roots in the same pot, and AhYSL1 expressed in the root epidermis showed transport activity for DMA-Fe in yeast. Recently, studies have implied the secretion of PS from tomato and grapevine, and the presence of endogenous DMA in the leaves and xylem of olive plants (Suzuki et al. 2016; Astolfi et al. 2020; Marastoni et al. 2020). Although the dependence of this on the Fe nutritional status has not been proven, these findings suggest that dicots also utilize exogenous and endogenous PS for the uptake and translocation of Fe. The direct uptake system is thought to be less sensitive to alkaline $\mathrm{pH}$ than the reduction-based uptake system, which requires acidification of the soil. Therefore, PDMA-Fe may be more useful for dicot species that highly depend on the direct uptake of PS-Fe. The possible direct uptake of PS-Fe by dicots should be investigated in the future.

The first study that compared the availability of PS-Fe and EDDHA-Fe in cucumber was by Römheld and Marschner (1986). In contrast to PDMA-Fe, the availability of PS-Fe was similar to or less than that of EDDHA-Fe. This discrepancy may be due to differences in the experimental design. In the previous study, PS secreted from Fe-deficient barley was supplied to the cucumber. Barley is known to secrete hydroxylated analogs of PS in addition to DMA (Takagi 1993; Ma et al. 1999). Although the capability of PDMA-Fe and DMA-Fe to be transported by YSL is similar (Suzuki et al. 2021), it is possible that the reducibility of PS-Fe is different between analogs, and the reducibility of PDMA-Fe could be equal to or higher than that of natural PS-Fe. For reduction-based uptake systems, highly reducible analogs can be a better Fe source. In fact, there is evidence that the synthetic microbial siderophore-Fe with high reducibility could provide more $\mathrm{Fe}$ than natural siderophore-Fe (Ueno et al. 2019).

In conclusion, PDMA-Fe can be utilized by cucumber roots more efficiently than traditional synthetic chelates in both calcareous substrate and 
hydroponic cultures. The pH-dependency tests showed that the higher availability of PDMA-Fe may be attributed to a higher reducibility at alkaline $\mathrm{pH}$. Our findings suggest that PDMA-Fe can be a good Fe fertilizer in alkaline soil for Strategy I plants.

Acknowledgements The authors thank Prof. Kosuke Namba from Tokushima University for the development and provision of PDMA, and Dr. Naoki Moritsuka from Kochi University for constructive comments on this research.

Author contribution D.U. and M.S. conceived and designed the study. D.U. and Y.I. performed the experiments. C.M., M.O., and T.S. contributed to the analysis of photosynthesis. All authors analyzed the data. D.U., C.M., and M.S. wrote the manuscript. All authors read and approved the manuscript.

Funding This work was financially supported by AICHI STEEL CORPORATION, Cabinet Office grant in aid, the Advanced Next-Generation Greenhouse Horticulture by IoP (Internet of Plants), Japan (grant number A12 to D.U.), and Core Research for Evolutional Science and Technology (CREST) of the Japan Science and Technology Agency, Japan (grant number JPMJCR1503 to C.M.).

Data availability Not applicable.

Code availability Not applicable.

\section{Declarations}

Ethics approval Not applicable.

Consent to participate Not applicable.

Consent for publication Not applicable.

Conflicts of interest/Competing interests M.S. is employed by AICHI STEEL CORPORATION. The remaining authors declare no competing interest.

Open Access This article is licensed under a Creative Commons Attribution 4.0 International License, which permits use, sharing, adaptation, distribution and reproduction in any medium or format, as long as you give appropriate credit to the original author(s) and the source, provide a link to the Creative Commons licence, and indicate if changes were made. The images or other third party material in this article are included in the article's Creative Commons licence, unless indicated otherwise in a credit line to the material. If material is not included in the article's Creative Commons licence and your intended use is not permitted by statutory regulation or exceeds the permitted use, you will need to obtain permission directly from the copyright holder. To view a copy of this licence, visit http://creativecommons.org/licenses/by/4.0/.

\section{References}

Ahmed E, Holmström SJ (2014) Siderophores in environmental research: roles and applications. Microb Biotechnol 7:196-208

Albano JP, Merhaut DJ (2012) Influence of FeEDDS, FeEDTA, FeDTPA, FeEDDHA, and $\mathrm{FeSO}_{4}$ on marigold growth and nutrition, and substrate and runoff chemistry. Horts 47:93-97

Astolfi S, Pii Y, Mimmo T et al (2020) Single and combined $\mathrm{Fe}$ and $\mathrm{S}$ deficiency differentially modulate root exudate composition in tomato: A double strategy for Fe acquisition? Int J Mol Sci 21:4038

Broadley M, Brown P, Cakmak I et al (2012) Function of nutrients: micronutrients. In: Marschner $\mathrm{P}$ et al (eds) Marschner's mineral nutrition of higher plants, 3rd edn. Academic, London, pp 191-248

Cesco S, Rombolà AD, Tagliavini M et al (2006) Phytosiderophores released by graminaceous species promote ${ }^{59} \mathrm{Fe}$-uptake in citrus. Plant Soil 287:223-233

Curie C, Panaviene Z, Loulergue C et al (2001) Maize yellow stripe 1 encodes a membrane protein directly involved in Fe(III) uptake. Nature 409:346-349

Eide D, Broderius M, Fett J, Guerinot ML (1996) A novel iron-regulated metal transporter from plants identified by functional expression in yeast. Proc Natl Acad Sci U S A 93:5624-5628

Gómez-Gallego M, Pellico D, Ramírez-López P et al (2005) Understanding of the mode of action of Fe(III)-EDDHA as iron chlorosis corrector based on its photochemical and redox behavior. Chemistry 11:5997-6005

Guerinot ML, Yi Y (1994) Iron: nutritious, noxious, and not readily available. Plant Physiol 104:815-820

Hasegawa H, Rahman MA, Saitou K et al (2011) Influence of chelating ligands on bioavailability and mobility of iron in plant growth media and their effect on radish growth. Environ Exp Bot 71:345-351

Hasegawa H, Rahman MM, Kadohashi K et al (2012) Significance of the concentration of chelating ligands on $\mathrm{Fe}^{3+}$-solubility, bioavailability, and uptake in rice plant. Plant Physiol Biochem 58:205-211

Hyvönen H, Orama M, Saarinen H, Aksela R (2003) Studies on biodegradable chelating ligands: complexation of iminodisuccinic acid (ISA) with $\mathrm{Cu}$ (II), $\mathrm{Zn}(\mathrm{II}), \mathrm{Mn}$ (II) and $\mathrm{Fe}(\mathrm{III})$ ions in aqueous solution. Green Chem 5:410-414

Inoue H, Kobayashi T, Nozoye T et al (2009) Rice OsYSL15 is an iron-regulated iron(III)-deoxymugineic acid transporter expressed in the roots and is essential for iron uptake in early growth of the seedlings. J Biol Chem 284:3470-3479

Jin CW, You GY, He YF et al (2007) Iron deficiency-induced secretion of phenolics facilitates the reutilization of root apoplastic iron in red clover. Plant Physiol 144:278-285

Lindsay WL, Schwab AP (1982) The chemistry of iron in soils and its availability to plants. J Plant Nutr 5:821-840

López-Rayo S, Hernández D, Lucena JJ (2009) Chemical evaluation of $\mathrm{HBED} / \mathrm{Fe}^{3+}$ and the novel $\mathrm{HJB} / \mathrm{Fe}^{3+}$ chelates as fertilizers to alleviate iron chlorosis. J Agric Food Chem 57:8504-8513 
López-Rayo S, Sanchis-Pérez I, Ferreira CMH, Lucena JJ (2019) [S,S]-EDDS/Fe: A new chelate for the environmentally sustainable correction of iron chlorosis in calcareous soil. Sci Total Environ 647:1508-1517

Lucena JJ (2006) Synthetic iron chelates to correct iron deficiency in plants. In: Barton LL, Abadia J (eds) Iron nutrition in plants and rhizospheric microorganisms. Springer, Dordrecht, pp 103-128

Ma JF, Nomoto K (1996) Effective regulation of iron acquisition in graminaceous plants. The role of mugineic acids as phytosiderophores. Physiol Plant 97:609-617

Ma R, Motekaitis RJ, Martell AE (1994) Stability of metal ion complexes of $N, N^{\prime}$-bis(2-hydroxybenzyl)ethylenediamine$N, N^{\prime}$-diacetic acid. Inorg Chim Acta 224:151-155

Ma JF, Taketa S, Chang YC et al (1999) Genes controlling hydroxylations of phytosiderophores are located on different chromosomes in barley (Hordeum vulgare L.). Planta 207:590-596

Ma JF, Ueno H, Ueno D et al (2003) Characterization of phytosiderophore secretion under Fe deficiency stress in Festuca rubra Plant Soil 56:131-137

Marastoni L, Lucini L, Miras-Moreno B et al (2020) Changes in physiological activities and root exudation profile of two grapevine rootstocks reveal common and specific strategies for Fe acquisition. Sci Rep 10:18839

Matsumoto K, Ozawa T, Jitsukawa K et al (2001) Crystal structure and redox behavior of a novel siderophore model system: a trihydroxamato-iron(III) complex with intraand interstrand hydrogen bonding networks. Inorg Chem 40:190-191

Morikawa CK, Saigusa M, Nakanishi H et al (2004) Co-situs application of controlled-release fertilizers to alleviate iron chlorosis of paddy rice grown in calcareous soil. Soil Sci Plant Nutr 50:1013-1021

Murata Y, Ma JF, Yamaji N et al (2006) A specific transporter for iron(III)-phytosiderophore in barley roots. Plant $\mathbf{J}$ 46:563-572

Nagata T, Oobo T, Aozasa O (2013) Efficacy of a bacterial siderophore, pyoverdine, to supply iron to Solanum lycopersicum plants. J Biosci Bioeng 115:686-690

Nowack B (2002) Environmental chemistry of aminopolycarboxylate chelating agents. Environ Sci Technol 36:4009-4016

Nowack B, Schwyzer I, Schulin R (2008) Uptake of Zn and Fe by wheat (Triticum aestivum var. Greina) and transfer to the grains in the presence of chelating agents (ethylenediaminedisuccinic acid and ethylenediaminetetraacetic acid). J Agric Food Chem 56:4643-4649

Nozoye T, Nagasaka S, Kobayashi T et al (2011) Phytosiderophore efflux transporters are crucial for iron acquisition in graminaceous plants. J Biol Chem 286:5446-5454

Pinto IS, Neto IF, Soares HM (2014) Biodegradable chelating agents for industrial, domestic, and agricultural applications-a review. Environ Sci Pollut Res Int 21:11893-11906

Romera FJ, Welch RM, Norvell WA, Schaefer SC (1996) Iron requirement for and effects of promoters and inhibitors of ethylene action on stimulation of $\mathrm{Fe}(\mathrm{III})$-chelate reductase in roots of strategy I species. BioMetals 9:45-50
Römheld V, Marschner H (1983) Mechanism of iron uptake by peanut plants: I. Fe reduction, chelate splitting, and release of phenolics. Plant Physiol 71:949-954

Römheld V, Marschner H (1986) Evidence for a specific uptake system for iron phytosiderophores in roots of grasses. Plant Physiol 80:175-180

Römheld V, Nikolic M (2007) "Iron". In: Barker AV (ed) Handbook of plant nutrition amnbnd Pilbeam. DJ CRC Press, Boca Raton, pp 351-374

Schenkeveld WDC, Hoffland E, Reichwein AM et al (2012) The biodegradability of EDDHA chelates under calcareous soil conditions. Geoderma 173-174:282-288

Sisó-Terraza P, Rios JJ, Abadía J et al (2016) Flavins secreted by roots of iron-deficient Beta vulgaris enable mining of ferric oxide via reductive mechanisms. New Phytol 209:733-745

Suzuki M, Nozoye T, Nagasaka S et al (2016) The detection of endogenous 2'-deoxymugineic acid in olives (Olea europaea L.) indicates the biosynthesis of mugineic acid family phytosiderophores in non-graminaceous plants. Soil Sci Plant Nutr 62:481-488

Suzuki M, Urabe A, Sasaki S et al (2021) Development of a mugineic acid family phytosiderophore analog as an iron fertilizer. Nat Commun 12:1558

Takagi S (1976) Naturally occurring iron-chelating compounds in oat- and rice-root washings. Soil Sci Plant Nutr 22:423-433

Takagi S (1993) Production of phytosiderophore. In: Barton LL, Hemming BC (eds) Iron chelation in plants and soil microorganisms. Academic Press Inc, San Diego, pp 111-131

Takagi S, Kamei S, Yu M-H (1988) Efficiency of iron extraction from soil by mugineic acid family phytosiderophores. J Plant Nutr 11:643-651

Tsai HH, Schmidt W (2017) Mobilization of iron by plantborne coumarins. Trends Plant Sci 22:538-548

Ueno D, Ma JF (2009) Secretion time of phytosiderophore differs in two perennial grasses and is controlled by temperature. Plant Soil 323:335-341

Ueno D, Rombolà AD, Iwashita T et al (2007) Identification of two novel phytosiderophores secreted by perennial grasses. New Phytol 174:304-310

Ueno D, Matsumoto K, Enami T et al (2019) Efficacy of an artificial microbial siderophore-Fe(III) with high redox potential on correcting Fe chlorosis in rice. Soil Sci Plant Nutr 65:471-478

Vansuyt G, Robin A, Briat JF et al (2007) Iron acquisition from Fe-pyoverdine by Arabidopsis thaliana Mol Plant Microbe Interact 20:441-447

Villén M, García-Arsuaga A, Lucena JJ (2007) Potential use of biodegradable chelate $N$-(1,2-dicarboxyethyl)-d,1aspartic acid/ $\mathrm{Fe}^{3+}$ as an Fe fertilizer. J Agric Food Chem 55:402-407

Waters BM, Lucena C, Romera FJ et al (2007) Ethylene involvement in the regulation of the $\mathrm{H}^{+}$-ATPase CsHAl gene and of the new isolated ferric reductase CsFRO1 and iron transporter CSIRT1 genes in cucumber plants. Plant Physiol Biochem 45:293-301

Xiong H, Kakei Y, Kobayashi T et al (2013) Molecular evidence for phytosiderophore-induced improvement of iron 
nutrition of peanut intercropped with maize in calcareous soil. Plant Cell Environ 36:1888-1902

Zuo Y, Zhang F, Li X, Cao Y (2000) Studies on the improvement in iron nutrition of peanut by intercropping with maize on a calcareous soil. Plant Soil 220:13-25
Publisher's note Springer Nature remains neutral with regard to jurisdictional claims in published maps and institutional affiliations. 